\title{
Role of reinsertion of the lower eyelid retractor on involutional entropion
} Roberto Caldato, Roberto Lauande-Pimentel, Nelson A Sabrosa, Roberto A Fonseca,
Raquel S Paiva, Milton R Alves, Newton K José University Medical School, UNICAMP, Campinas, São Paulo, Brazil

R Caldato

R Lauande-Pimentel

N Sabrosa

R Fonseca

R Paiva

M Alves

N Kara José

Department of Ophthalmology,

University of São

Paulo Medical School,

USP, São Paulo, Brazil

R Lauande-Pimentel

$R$ Fonseca

R Paiva

M Alves

N Kara José

Correspondence to: Roberto Caldato, MD, Rua Coronel Quirino, 1615, Ed Parthenon, ap 131,

Campinas-SP, Brazil, CEP 13025-002

caldato@correionet.com.br

Accepted for publication 15 June 1999

Eyelid entropion is a condition in which the margin of the eyelid is inverted against the corneal and conjunctival surface, causing damage to these structures as a result of eyelash and skin friction. Hyperaemia, foreign body sensation, lacrimation, and vision loss are concurrent complaints. Secondary infection may occur. Several surgical procedures have been described in the past years to solve this problem. Recurrence has been reported with all these procedures. ${ }^{1}$ The surgical techniques were based on the assumption that the pathophysiology of this disease is due to the following factors: (1) enophthalmos, (2) horizontal laxity of the inferior eyelid, (3) anomalous action of the preseptal orbicular muscle, overriding the pretarsal orbicular muscle, and (4) dehiscence or disinsertion of lower eyelid retractors. ${ }^{2}$

Jones $e t a l^{34}$ described for the first time the role of the lower eyelid retractors in involutional entropion pathophysiology and during the intervening years researchers have paid great attention to this finding. De Roeth ${ }^{5}$ emphasised the fact that the involutional

\begin{abstract}
Department of Ophthalmology, Campinas State

Abstract

Aims-To verify and evaluate the effect of reinsertion of the lower eyelid retractor aponeurosis to correct involutional entropion.

Methods-The involutional entropion is one affection that occurs mainly in the lower eyelid of patients over 60 years old. The surgical techniques proposed to correct this condition are based on correction of horizontal laxity-the preseptal orbicularis muscle overrides the pretarsal muscle, and the reinsertion of the lower eyelid retractor aponeurosis. 30 patients clinically diagnosed with involutional entropion and randomly selected underwent reinsertion of the lower eyelid retractor aponeurosis to the tarsal plate, without horizontal shortening or resection of the skin or orbicularis muscle.

Results-Good anatomical and functional correction was achieved in $\mathbf{9 6 . 6 \%}$ of the patients and no recurrence was observed on 29 month follow up examination. The surgical result was very satisfactory.

Conclusions-It was concluded that this procedure is effective and has low recurrence rate, showing the important role of the reinsertion of the lower eyelid retractor aponeurosis in this surgical correction.

(Br F Ophthalmol 2000;84:606-608)
\end{abstract}

entropion is temporarily corrected after instillation of adrenergic drops (cocaine 5\%) in the inferior conjunctival sac, stimulating the inferior tarsal muscle.

An analogy of what occurs in the superior eyelid, where correction of the involutional ptosis is made by reinsertion of the superior eyelid retractor to the tarsal plate, we propose a similar surgical approach to correct involutional entropion, by means of reinserting the inferior eyelid retractor to the tarsal plate, without any other procedure such as horizontal shortening of the eyelid or approaching the orbicularis muscle. To the best of our knowledge, this study is the first to evaluate the surgical reinsertion of the retractor aponeurosis.

\section{Patients and methods}

We evaluated, from March 1994 until September 1995, all patients referred to the oculoplastic department at UNICAMP with the initial diagnosis of entropion. We excluded from the study all cases not characterised as involutional - that is, cicatricial or congenital entropion. In the case in which patients presented with bilateral disease, we included, randomly, only one of the eyes. Information concerning demographic data was gathered from each case (name, age, race, sex) and associated ophthalmic and systemic diseases were noted (Fig 1).

After thorough cleaning and draping of the eyelid we performed infiltration of the skin with an anaesthetic solution of xylocaine $2 \%$ with adrenaline $(1: 200000)$. Subciliary incision, as in the blepharoplasties, was performed through the skin and a 4-0 silk suture was placed at the superior edge of the incision, to keep a slight superior traction on the eyelid. We then dissected the pretarsal orbicularis muscle until the tarsal plate was exposed and continued inferiorly to the orbital septum. Haemostasis with electrocautery was used when necessary. After slight pressure over the globe, we

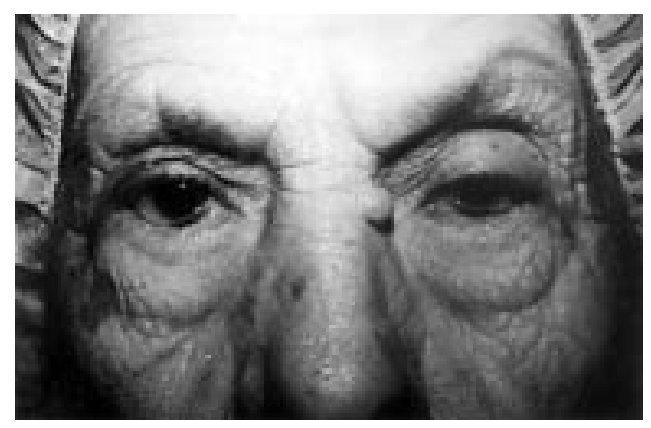

Figure 1 Patient selected for surgical procedure. 


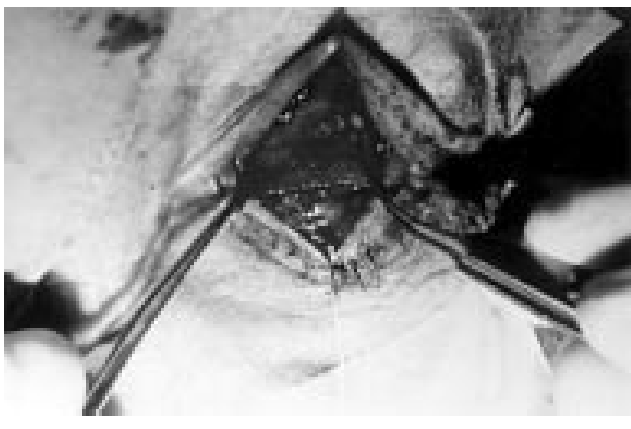

Figure 2 Identification of aponeurosis was confirmed by holding it with forceps and asking the patient to look downwards.

detected the orbital fat pad, and over it the septum was opened. Moving this structure inferiorly with a cotton tip soaked in saline solution, we identified the retractor aponeurosis, according to its characteristic amber surface. Identification of the aponeurosis was confirmed by holding it with forceps and asking the patient to look downwards (Fig 2). In the positive case, traction could be felt as the muscle contracted.

The reinsertion of the retractor to the edge of the lower tarsus was done with 6-0 silk. A first horizontal double mattress suture was placed on a vertical line that passed over the pupillary centre, when the patient was looking in primary position of gaze. It was not possible to distinguish at surgery if the patient presented a total disinsertion or only retractor lengthening. The first horizontal double mattress suture was placed on the aponeurosis as low as possible, to avoid making further entropion or retraction of the lower eyelid.

A good excursion of the lower eyelid could be observed at this point by asking the patient to look up and down. Once the eyelid had a satisfactory positioning, two other separate double mattress sutures were placed on either side of the first one. Further, the skin was sutured with $6-0$ silk. The eye was occluded with antibiotic ointment.

Postoperatively the routine was: (1) next day the patient returned to have the drapes remove and local cleansing was carried out; (2) at 1 week they returned to have the skin sutures removed; (3) after 1 month they returned for functional evaluation; and (4) they returned every 3 months for standard follow up (Fig 3).

We defined surgical success as the disappearance of symptoms and good positioning of the eyelid margin, on apposition to the globe in

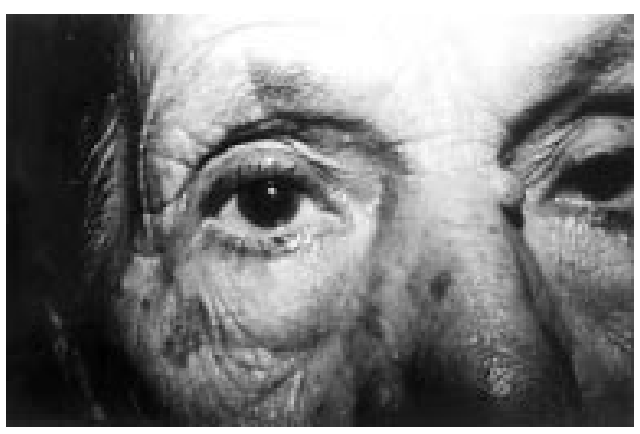

Figure 3 Follow up visit, with good positioning of the eyelid margin. primary position of gaze, when looking down or blinking. We considered as recurrence of entropion the appearance of a new inversion of the lower eyelid edge after surgical correction, when in primary position of gaze, infraversion, or blinking. Early and delayed recurrence were defined when occurring before and after 18 months respectively.

\section{Results}

Sixty two patients were diagnosed as having senile entropion during the period March 1994 through September 1995. From this group of patients, 30 were randomly selected to undergo the surgical procedure. Hence, we studied 30 eyes of 30 patients with clinical diagnosis of involutional entropion; 15 right eyes, and 15 left eyes were used in the study. Fourteen patients were male and 16 were female. The mean age was 71.6 (SD 11) years. All these patients underwent the outpatient surgical procedure, without complications. The follow up period varied from 19 to 36 months postoperatively, with an average of 28.7 months. We did not notice early or delayed recurrences during our follow up.

We achieved surgical success in $96.6 \%$ of the cases. The only case considered unsuccessful was a 74 year old man, who presented an excessive horizontal laxity of the eyelid. $\mathrm{He}$ developed an early postoperative ectropion of the lower eyelid. We decided to correct it in the sixth month of follow up with a horizontal shortening of the inferior eyelid. The surgical result turned out to be very satisfactory.

\section{Discussion}

Several techniques were described for the correction of involutional entropion. Jones et $a l^{4}$ noted that between 80 and 200 procedures have already been mentioned in the medical literature. The great variation of techniques suggests that there is not an easy way to solve the problem. The orbicularis muscle is anatomically divided into preseptal and pretarsal parts. The first lies over the tarsus and the second over the septum. The pretarsal muscle is adheres firmly to the tarsus and the preseptal muscle is weakly adherent to the septum. The involution makes these connections even weaker. The orbital septum extends from the orbital rim to the inferior edge of the tarsus. Below the septum, one can find the orbital fat pad and behind it the aponeurosis of the eyelid retractor.

The inferior eyelid retractor is responsible for the tarsal plate stability and for excursion of 3-5 $\mathrm{mm}$ of the inferior eyelid when in downward gaze. The motor components of the retractors are the inferior rectus. An expansion of the inferior rectus capsule extends anteriorly, involves the inferior oblique muscle, and goes to the inferior edge of the tarsal plate. A small band of smooth muscle inserts also on the inferior edge of the tarsus. It originates from the tissues that involve the inferior oblique muscle. This muscle is similar to the superior eyelid Muller's muscle and is called the inferior tarsal muscle. Its innervation is made by the sympathetic nerve. Some fibres 
insert at the conjunctival sac, which is pulled inferiorly when in downward gaze. The anatomy of the inferior eyelid retractors is similar to that of the superior's eyelid elevator complex. ${ }^{4}$

The inferior eyelid retractors become thinner and longer with aging. In some cases, dehiscence or disinsertion of its connection to the tarsus can occur. These anatomical alterations lead to a decrease of the vertical eyelid support making it more mobile. ${ }^{3}$

The absorption of the fat pad also occurs with ageing, causing enophthalmus. This leads to a decrease in globe pressure against the eyelid. The overlying skin loses tension, becomes redundant and less adherent to the orbicularis muscle. The preseptal portion of orbicularis muscle becomes less adherent to the septum and, when blinking, slides superiorly between the overlying skin and the pretarsal orbicularis muscle. Since in this study no subciliary incision was done, as in blepharoplasties, we did not make a barrier to allow the preseptal muscle to override.

The thinning of eyelid ligaments reduces horizontal support of the eyelid and entropion may occur under the following conditions: (1) the retractor does not pull the eyelid down and backwards; (2) enophthalmus occurs because of fat pad absorption, leading to a decrease in contact of the globe with the inferior eyelid; (3) the laxity of the eyelid ligaments decreases horizontal support of the eyelid; (4) the preseptal orbicularis muscle, weakly adherent to the septum, overrides the skin and pretarsal orbicularis turning them inwards. Under these conditions the eyelid margin turns inwards causing entropion.

Jones $^{3}$ called attention to, and described, the fact that involutional entropion was related to malfunction of the inferior eyelid retractors. In past years, all techniques described have tried to correct the retractors, the preseptal orbicularis, and the eyelid's horizontal laxity. Similar involutional alterations in the superior eyelids lead to ptosis, which is solved with reinsertion of superior eyelid's aponeurosis.

In the procedure described in this article there was no correction of horizontal laxity, no resection of preseptal orbicularis muscle, and no creation of cicatricial barrier to block preseptal orbicularis muscle override of pretarsal orbicularis muscle. Hence, we approached this disease in a analogous way to that of an involutional ptosis, since both have similar pathophysiological features. Our results were very satisfactory, since the success rate was $96.6 \%$ of the studied population and we have not detected any recurrences during our follow up period.

The correction of disinsertion of the inferior eyelid retractor's aponeurosis as an isolated procedure proved to be effective in the correction of involutional entropion. The results we obtained with this procedure suggests that the aponeurosis dehiscence or disinsertion may be the most important factor leading to this disease.

We wish to acknowledge the help of Mr JRO Collin, consultant ophthalmic surgeon, Moorfields Eye Hospital, for interesting comments.

1 Benger RS, Barthey R. Involution entropion: a review of the management. Ophthalmic Surg 1987;18:140-2.

2 Drydem RM, Leibsoh J, Wobig JL. Senile entropion: pathogenesis and treatment. Arch Ophthalmol 1987;96: 1883

3 Jones LT. The anatomy of the lower eyelid and its relation to the cause and cure of entropion. Am F Ophthalmol 1972;74: 327.

4 Jones LT, Reeh MJ, Wobig JL. Senile entropion: a new concept for correction. Am f Ophthalmol 1972;74:327-9.

5 De Roeth AF. Mechanism of senile entropion. Trans Pacific Coast Oto-Ophth Soc 1963;44:173. 